\title{
Sustainability in the information society
}

\section{Conference Paper}

Author(s):

Köhler, Annette; Loerincik, Yves; Hertwich, Edgar; Jolliet, Olivier

Publication date:

2004

Permanent link:

https://doi.org/10.3929/ethz-b-000050508

Rights / license:

In Copyright - Non-Commercial Use Permitted

Originally published in:

The International Journal of Life Cycle Assessment 9(3), https://doi.org/10.1007/BF02994197 


\title{
State-of-the-Art in LCA
}

\section{Sustainability in the Information Society}

\section{1th SETAC Europe LCA Case Studies Symposium}

3-4 December 2003

\author{
Annette Köhler ${ }^{1^{*}}$, Yves Loerincik ${ }^{2}$, Edgar Hertwich ${ }^{3}$ and Olivier Jolliet ${ }^{2}$ \\ ${ }^{1}$ Swiss Federal Institute of Technology, Zurich, Safety and Environmental Technology Group, ETH-Hoenggerberg HCI, \\ $\mathrm{CH} 8093$ Zurich, Switzerland \\ ${ }^{2}$ Ecole Polytechnique Fédérale de Lausanne, Laboratory of Ecosystem Management, Industrial Ecology and Life Cycle Systems, EPFL, \\ CH-1015 Lausanne, Switzerland \\ ${ }^{3}$ Norwegian University of Science and Technology, Department of Energy and Process Engineering, Program for Industrial Ecology, \\ K. Hejes vei 1a, NO-7491 Trondheim, Norway
}

* Corresponding author (annette.koehler@chem.ethz.ch)

The 11th SETAC Europe LCA Case Studies Symposium was heid at the Swiss Federal Institute of Technology Lausanne (EPFL), Switzerland on 34 December 2003, in conjunction with the International Society for Industrial Ecology and the $21^{\text {st }}$ Swiss Discussion Forum. Organised by the Industrial Ecology and Life Cycle Systems group of EPFL, this meeting addressed a number of interesting issues, including the environmental impacts of information and communication technology $(I C T)$, the use of ICT to avoid environmental impacts, the sensibility of product policies regarding ICT, the rebound effect, and the application of ICT in environmental assessment.

\section{New Materials for ICT}

As the electronic waste recycling has been introduced in the European legislation, manufacturers have to organise the take-back and recycling of electronic waste. Thus, incentive is given for the development of new materials, partly based on renewable resources. New materials are increasingly used and constitute interesting progress concerning application in various areas. This was the focus of the first session.

MARTIN MÖLler compared a newly developed printed circuit board (PCB) based on foamed high temperature thermoplastics (HTT) with the conventional PCB containing a flame retardant. The results presented show that the HTT $\mathrm{PCB}$ is environmentally preferable to the conventional PCB, due to a higher resource efficiency, a more efficient production and a lower hazardous substance potential.

Addressing the challenges of mobility and transport, GERALD REBITZER presented a detailed LCA study of a new aluminium honeycomb material developed, produced, and marketed by Alcan. He demonstrated that the application of the honeycomb material, e.g. in passenger automobiles, leads to significant environmental savings.

The presentation by MARC-ANDRE WOLF gave insight into the results of a case study comparing conventional lead-containing and various new, lead-free solder-pastes. He suggested that, following an LCA view, lead-free solder can be an improvement. However, this is not necessarily the case. The toxicological risks from fire hazard and inappropriate recycling cannot adequately be addressed by LCA.

JOSEF KÄNZIG presented the possible use of bio-based products in the field of ICT. Lignin can be used for Printed Wiring Boards (PWB), for instance, and bio-based ink has made some progress in application during the last years.

In the general discussion of this session, the question was raised whether the studies presented were compliant with the ISO 14040 series standards since they compared different product alternatives, but did not include critical reviews as required for comparative assertions disclosed to the public. Although no complete consensus could be reached, it was concluded that one should differentiate between comparative assertions disclosed to the public, e.g. for marketing purposes, and published/presented product comparison for internal and reference purposes, without the intention to make any claims. It was proposed that this is an issue which should be clarified in the ongoing revision of the ISO $14040-14043$.

\section{ICT Infrastructure}

The infrastructure is the basic prerequisite to appropriately run ICT. As the number of equipment devices is likely to heavily increase in the coming years, it is necessary to understand where the main environmental impacts stem from and to find solutions to limit them. This was the subject of the second session.

Impacts of electromagnetic radiation (EM) were the focus of the presentation by ALFRED BÜRGI. He introduced simple model calculations of the field strength of EM due to base stations for the $2 \mathrm{G}$ network in the city of Bern. As field measurements had not been conducted and most data are confidential data of the network operators, a precise modelling of the radiation exposure cannot be performed. Thus, even if toxicological tests, such as those presented by RAINER FRENTZEL-BEYME (poster session), provide possible dose-response relationships, a risk estimate or an impact assessment is hardly feasible.

JENS MALMODIN documented a peer-reviewed LCA of the infrastructure of a third generation of mobile telephones (3G) for a net with 1.5 million subscribers and 3000 base stations. He showed that, per subscriber and year, the network consumes $100 \mathrm{kWh}$ of energy, and causes $55 \mathrm{~kg}$ of $\mathrm{CO}_{2}$ emissions and $6.7 \mathrm{~kg}$ of solid waste. As the emissions correspond to less than $1 \%$ of the typical per capita $\mathrm{CO}_{2}$ emissions in industrialised countries, MALMODIN advocated further promotion of $3 \mathrm{G}$ technology due to its benefits to society. 
ICT services using the Internet Protocol (IP) network are considered to produce fewer environmental burdens than the services using traditional PSTN due to the simpler structure of IP networks. KaTSUHIKO HoNjo presented an evaluation of the environmental impacts of the IP network connection service by means of life cycle inventory analysis. He demonstrated that $\mathrm{CO}_{2}$ emissions of the IP network connection service provided by DSL (digital subscriber line) or FTTH (fibre to the home) connection are less than emissions caused by the service provided by dial-up connection using PSTN.

TOMOHKO FURUTANI introduced a dynamic model for analysing the environmental impacts of Internet infrastructure for broadband access in Japan. By integrating a population balanced model into LCA, the production of electronic devices and their electricity consumption during use were evaluated in terms of $\mathrm{CO}_{2}$ emissions. The results indicated that $\mathrm{CO}_{2}$ emissions are likely to increase by a factor of 2 from 2002 to 2005 due to growing electricity demand and fabrication of new devices.

HEIDI HoTTENROTH compared a wired and wireless local area network (LAN and WLAN). Her presentation showed that there is little difference between the two types of networks for household operations, while the WLAN has significantly lower emissions of greenhouse gases, acidifying gases, and photooxidants for a small office with 20 PCs.

Other presenters analysed the environmental consequences of end-of-life scenarios for ICT. KARSTEN SCHISCHKE stated that the reuse of PCs ranks notably better in terms of ecological performance than material recycling due to the complexity of electronics. Also, the eco-efficiency of PC use is increased by reuse.

ROLAND HISCHER elaborated on the environmental impacts of the Swiss electronic scrap recycling system (SWICO) and came to the conclusion that the benefit from material recovery in SWICO outweighs the environmental load caused by the system.

The following discussions focused on the key issues that should be addressed in future investigations such as the implications for policy making and the improvement of knowledge on consumer behaviour and use patterns. As most of the studies applied only standard impact categories, e.g. cumulative energy demand and global warming, the lack of considering ecosystem and human health and electromagnetic radiation was heavily debated.

\section{Indirect Effects}

One key question when studying the environmental impacts of ICT involves the indirect effects. How will the society evolve with ICT? What are the rebound effect links to Ecommerce and other E-services? These questions were addressed in the session on Indirect Effects.

The objective of the study performed by SANGWON SUH was to better understand the complex interplay between technology, composition and scale of the information sector in order to estimate the material and energy terms of information society and to envisage a desirable industrial metabolic structure for the future. SuH showed that the future information society will be characterised by a higher information density, a more efficient technology and a higher share of the in- formation industry in the industrial sectors' composition. In the future, the transmission of information will lead to less environmental impacts, although the exponential growth in the amount of information will outweigh those gains.

The implications of ICT on consumption and the rebound effect were addressed by EDGAR HERTWICH who provided a classification scheme of the rebound effect and an analytical framework for measuring several impacts of the entire consumption basket. As ICT is a transformational technology, HeRTWICH proposed the term 'transformational effect' instead of the term 'rebound effect'. ICT plays a major role for economic growth and, therefore, induces an increase of emissions from indirect effects.

LORENZ HILTY presented a system dynamics model considering dematerialization and rebound effects with less resolution on the process level than a conventional LCA. This session's presentations triggered a debate on the question of scenario analysis and a better clarification of terminology from different fields as the rebound effect.

'Too much information kills the information' was the starting point of the discussion. It was proposed to define a unit of 'useful information'. The calculation of the environmental impact per unit of 'useful information' could be a better indicator of the sustainability of the information society. It was also noted that indirect effects, such as rebound effects, should be systematically taken into account in the studies on ICT. Finally, the question of the value of rebound effect studies was raised.

\section{Dematerialization}

Dematerialization is a controversial topic and its potentials are difficult to estimate. Among other developments, software are delivered over the Internet, MP3 music format tends to replace the audio $\mathrm{CD}$, dictionaries can be found on the Internet. The next session focused on the question whether the environmental effects of such a technological progress will be important.

THOMAs KöLLNER described how Input-Output LCA can be used for evaluating the portfolios of about 25 investment funds. He especially investigated how the constructed fund portfolios deviate from their financial benchmark and how this affects the overall environmental impact of these funds. The general discussion raised the issue of potential negative effects that could occur in case of deinvestments in economic sectors with high environmental impacts.

Are Norwegian enterprises concerned about the fact that service activities also give rise to environmental impacts by travelling, heating, using technical equipment like PCs etc? INGUNN SAUR-MODHAL stated in his presentation that service enterprises are more concerned about service activities than the traditional industry is. Nevertheless, in production facilities up to $50 \%$ of the environmental impacts can also have their source in service activities. By analysing one company SAUR MODHAL indicated that business travels had the biggest contribution to environmental impacts in terms of energy consumption and $\mathrm{CO}_{2}$ emissions. Consumption of electricity for offices and the use of office equipment were also important for energy consumption. In addition, commuting to work made up for a significant contribution to environmental burdens. 
Describing a longitudinal study, LORENZO VICARIO showed that the adoption of digital music brings about several new possibilities such as transferring or sharing pieces of music through the Internet. However, this service also has some negative effects including lower quality and some usability problems. VICARIO's results suggest that the different, nonoverlapping features of the two services (conventional and digital music) do not favour a complete replacement of the first with the second. Unlike the digital music, the service provided by E-mails is recognised to cover most of the functionalities and features offered by conventional letters, having additional benefits such as prompt delivery and low costs. Thus, the degree of replacement tends to be higher.

JEAN-MARC TEMERSON described various new emerging services such as a telemedicine service. In the field of education and culture, he demonstrated that virtual visits, for example, could promote the visit of museums while avoiding transportation to the location, whereas new technologies are bringing Mobile Imaging services to professionals in the teleactivities field.

Finally, a case study comparing traditional newspaper delivery with electronic information delivery via Internet was presented by HIROSHI YaGTTA. As for net-delivery type newspapers, it was confirmed that $\mathrm{CO}_{2}$ emissions vary according to the kind of equipment device used for browsing, the browsing behaviour and the frequency of printing the information.

\section{ICT Applications}

E-commerce has strongly developed during the last years. The environmental impacts of this type of services have been studied, while the environmental benefits do not seem obvious. The session on ICT applications was devoted to the question whether new types of impacts are replacing those due to traditional commerce or whether E-commerce performs environmentally friendlier.

SAJED ABUKHADER stated that nowadays there is a need for assessing a list of strategies related to the management of supply chains and logistics discipline, such as postponement, customisation, centralisation/decentralisation, etc. One situation of importance, where this is apparent and also crucial, is the E-commerce issue. This shows why LCA seems to be of insufficient ability to assess the environmental implications of E-commerce at the current stage.

JIRO NAKAMURA gave insight into his modelling results of different scenarios of E-commerce in Japan, that included benefits from reduced warehousing, reduced wholesale trade, and reduced transport to retail stores. By 2010 , the quantified ICT net benefits would amount to $3.9 \%$ of Japan's energy consumption. His presentation showed that significant reductions in $\mathrm{CO}_{2}$ emissions could be expected from E-commerce. The following discussion focused on the fact that indirect effects were not taken into account and that they could play a very important role.

ERIC WILIJAMs demonstrated a remarkable reduction potential of energy-related environmental burdens through telecommuting. Results are that adoption of 4-day per week telecommuting of the mobile sales and specialist/technical work force could reduce national energy consumption by $1.0 \%$ in Japan and by $0.4 \%$ in the US. While transport savings are the dominant factor in the energy balance of telecommuting, changes in energy use in buildings, both commercial and residential, are significant and deserve attention.

The city of Martigny in Switzerland set up a telemonitoring network to enhance the management of various industrial networks such as the water, gas, electricity, and district heating, which were investigated by YVES LOERINCTK. He applied LCA to show that the gain in management efficiency by telemonitoring overcomes the environmental impact of the infrastructure, showing that ICT can have a positive effect on the environment.

Working in the West Bengal Jute Project (WBJP) that intends to diversify the jute market, MARGARTTHA OsSEs elaborated on new GIS-based tools which are applied to monitor and manage both agricultural practices and the post-harvest retting process. OSsEs explained that the introduction of such an ICT-based monitoring system has the potential to significantly alter the traditional value chain in the jute market. Some of the resulting effects might be positive for the environment. However, a more efficient value chain might also increase the general demand for jute, finally leading to environmental rebound effects.

Llorenç Mra i Canals described the elCA pan-European project as an example of ICT applications. Small- and Mediumsized Enterprises (SMEs) provide the majority of economic activity across Europe, but are unlikely to implement integrated product policy because of the high costs of gathering data as well as the knowledge and resources to manage this information. The project brings together LCA and ICT by providing a web portal (ecosmes.net), containing pre-compiled life cycle data linked to a simplified life cycle analysis tool.

Thus, a lively discussion followed on ICT net benefits, as there was common agreement that side effects on E-commerce have to be better examined in future investigations. Also, a number of issues was raised with respect to the evaluation of the overall environmental consequences and the optimisation of environmental benefits.

\section{Conclusion}

The presentations and the discussions show that significant progress has been achieved in the field of studying environmental impacts of ICT, while further efforts are required in several domains.

Within the last years, methodological extensions of LCA and similar methods were made that enable one to take into account not only hardware but also software and service-based developments. While LCA and related assessment tools are appropriate to identify the ICT key factors responsible for significant environmental impacts, the discussion identified deficiencies in the consideration of two important categories: human and ecotoxicity, and electromagnetic radiation. These environmental effects still deserve intensive investigations in order to improve the overall environmental assessment of ICT.

One of the main conference outputs was that data sharing needs to be intensified, both for hardware as well as for software and service applications. Data quality was recognised as the most important basis for the reliability of LCA results, especially in a field such as ICT. In addition, it was emphasised in the general debate that indirect and rebound effects could have a tremendous environmental impact. Efforts to assess these effects as well as the changes in consumption patterns due to ICT should be pursued and more deeply studied.

The best presentation award went to SANGWON SUH of Leiden University for his work on information computation. The best poster award was given to MIREILLE FAIST of ESU Services for her LCA of the 3G mobile communication system. 